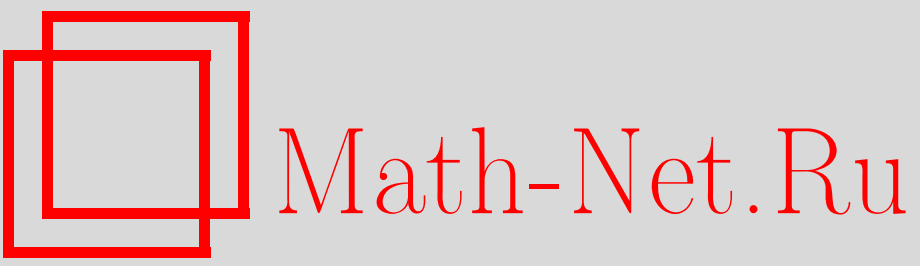

И. Лорис, Билинейные представления интегрируемых уравнений, ТМФ, 2002, том 133, номер 2, 270-278

DOI: https://doi.org/10.4213/tmf396

Использование Общероссийского математического портала Math-Net.Ru подразумевает, что вы прочитали и согласны с пользовательским соглашением

http: //www.mathnet.ru/rus/agreement

Параметры загрузки:

IP : 54.224 .187 .69

26 апреля 2023 г., 12:54:43 


\section{БИЛИНЕЙНЫЕ ПРЕДСТАВЛЕНИЯ ИНТЕГРИРУЕМЫХ УРАВНЕНИЙ}

Предложен метод, позволяющий получать операторы рекурсии и канонические пары Лакса непосредственно из билинейных тождеств типа Кадомцева-Петвиашвили. Приведенные примеры включают в себя уравнение Кортвега-де Фриза, уравнение Буссинеска и вещественный аналог нелинейного уравнения Шредингера.

Ключевые слова: солитон, симметрия, интегрируемость, нелинейное дифференциальное уравнение.

\section{1. ВВЕДЕНИЕ}

Для интегрируемых уравнений в частных производных, таких как уравнения Кортевега-де Фриза (КдФ) [1] и Буссинеска [2] (которые были изначально получены для описания волн на поверхности воды, но потом нашли гораздо более широкое физическое применение [3]), характерно наличие бесконечного числа симметрий и законов сохранения. Эти симметрии старших порядков (связанные с дополнительными переменными времени) могут интерпретироваться как потоки совместных нелинейных уравнений в частных производных (НУрЧП) старших порядков. Интегрируемые НУрЧП образуют так называемые иерархии [4]. Оператор рекурсии (для некоторого уравнения) отображает симметрии в симметрии; его (формально) сопряженный оператор можно использовать для того, чтобы породить бесконечный набор законов сохранения. В данной работе будет описан метод, позволяющий получать такие операторы рекурсии.

Метод Хироты [5] представляет собой мошный метод исследования солитонных уравнений. Он основывается на билинейных представлениях и использовании фундаментальных полевых переменных, представляющих собой не что иное, как тау-функции, и дифференциальных операторов Хироты $D_{x}$ :

$$
D_{x} f \cdot g=f_{x} g-f g_{x}, \quad D_{x}^{2} f \cdot g=f_{2 x} g-2 f_{x} g_{x}+f g_{2 x}
$$

и т.д. (нижние индексы обозначают производные, $f_{n x}:=\partial_{x}^{n} f$ ). Техника Хироты сводится к использованию так называемых билинейных тождеств (см. раздел 2 ) для иерархии Кадомцева-Петвиашвили (КП). Собственно уравнение КП имеет вид [6]

$$
4 u_{x, t_{3}}-3 u_{2 t_{2}}-u_{4 x}-6\left(u u_{x}\right)_{x}=0 .
$$

\footnotetext{
*Dienst Theoretische Natuurkunde Vrije Universiteit Brussel, Brussel, Belgium
} 
Связь между формализмом Хироты и операторами рекурсии была детально изучена в работе [7]. В данной работе будет показано, как установить такую связь в случае билинейных тождеств для иерархий уравнения КП и модифицированного уравнения КП.

\section{2. БИЛИНЕЙНЫЕ ТОЖДЕСТВА}

Иерархия КП интегрируемых $(2+1)$-мерных НУрЧП задается условиями совместности $[8]$

$$
\frac{\partial B_{m}}{\partial t_{n}}-\frac{\partial B_{n}}{\partial t_{m}}=\left[B_{n}, B_{m}\right]
$$

(для любых $n, m$ ) системы линейных уравнений

$$
\Phi_{t_{n}}=B_{n} \Phi, \quad n=2,3, \ldots
$$

т.е.

$$
\Phi_{t_{2}}=\Phi_{2 x}+u \Phi, \quad \Phi_{t_{3}}=\Phi_{3 x}+\frac{3}{2} u \Phi_{x}+3\left(\frac{1}{2} u_{x}+u_{3}\right) \Phi
$$

и т.д. Эти НУрЧП для полей $u(\underline{t}), u_{3}(\underline{t}), \ldots$, где $\underline{t}=\left(t_{1}=x, t_{2}, t_{3}, \ldots\right)$, можно записать в компактном виде после преобразования переменных следуюшего вида (билинеаризующего преобразования):

$$
u=2 \partial_{x}^{2} \ln \tau, \quad u_{3}=\frac{1}{2}\left(\partial_{t_{2}}-\partial_{x}^{2}\right) \partial_{x} \ln \tau, \ldots
$$

Тау-функция $\tau$ удовлетворяет следуюшему соотношению (билинейному тождеству КП) $[8]$

$$
\operatorname{Res}_{\lambda}\left[\tau(\underline{t}-\underline{\epsilon}(\lambda)) \tau\left(\underline{t}^{\prime}+\underline{\epsilon}(\lambda)\right) e^{\xi\left(\underline{t}-\underline{t}^{\prime}, \lambda\right)}\right]=0
$$

для любых $\underline{t}, \underline{t}^{\prime}$. Здесь $\operatorname{Res}_{\lambda}$ есть коэффиициент при $\lambda^{-1}, \underline{\epsilon}(\lambda)=\left(\lambda^{-1}, \lambda^{-2} / 2, \lambda^{-3} / 3, \ldots\right)$, а $\xi(\underline{t}, \lambda)=\lambda t_{1}+\lambda^{2} t_{2}+\lambda^{3} t_{3}+\cdots$. Это тождество порождает бесконечный набор уравнений в частных производных на поле $\tau$. Прямо вычисляя коэффициент при $\lambda^{-1}$ в формуле (7), получим уравнение КП в билинейной форме Хироты. Следуя работе [8], найдем (после замены переменных $\underline{t}=\underline{x}-\underline{y}, \underline{t}^{\prime}=\underline{x}+\underline{y}$ )

$$
\operatorname{Res}_{\lambda}\left[\tau(\underline{x}-\underline{y}-\epsilon(\lambda)) \tau(\underline{x}+\underline{y}+\epsilon(\lambda)) e^{-2 \xi(\underline{y}, \lambda)}\right]=0,
$$

или

$$
\operatorname{Res}_{\lambda}\left[e^{\xi\left(\tilde{\partial}_{y}, \lambda^{-1}\right)}(\tau(\underline{x}-\underline{y}) \tau(\underline{x}+\underline{y})) e^{-2 \xi(\underline{y}, \lambda)}\right]=0 .
$$

Используя определение

$$
e^{\xi(\underline{t}, \lambda)}=\sum_{n \geqslant 0} \lambda^{n} p_{n}(\underline{t})
$$

многочленов Шура $p_{n}(\underline{t})$, можно привести (9) к виду

$$
\sum_{j=0}^{\infty} p_{j}(-2 \underline{y}) p_{j+1}\left(\tilde{\partial}_{y}\right) \tau(\underline{x}-\underline{y}) \tau(\underline{x}+\underline{y})=0
$$


для любого $\underline{y}$, или же с использованием оператора Хироты $\widetilde{D} \equiv\left(D_{1}, D_{2} / 2, D_{3} / 3, \ldots\right)$, где $D_{n}-D$-оператор Хироты по отношению к времени $t_{n}($ см. определение $(1))$, получим

$$
\sum_{j=0}^{\infty} p_{j}(-2 \underline{y}) p_{j+1}(\widetilde{D}) \exp \left(\sum_{i=1}^{\infty} y_{i} D_{i}\right) \tau \cdot \tau=0
$$

для любого $\underline{y}$. Низшие нетривиальные уравнения из набора (11) имеют вид:

$$
\begin{aligned}
\left(4 D_{1} D_{3}-3 D_{2}^{2}-D_{1}^{4}\right) \tau \cdot \tau & =0, \\
\left(3 D_{1} D_{4}-D_{2} D_{1}^{3}-2 D_{2} D_{3}\right) \tau \cdot \tau & =0 \\
\left(144 D_{1} D_{5}-45 D_{1}^{2} D_{2}^{2}-20 D_{1}^{3} D_{3}-80 D_{3}^{2}+D_{1}^{6}\right) \tau \cdot \tau & =0 \\
\left(36 D_{2} D_{4}+4 D_{1}^{3} D_{3}-32 D_{3}^{2}-9 D_{1}^{2} D_{2}^{2}+D_{1}^{6}\right) \tau \cdot \tau & =0, \quad \ldots
\end{aligned}
$$

Первое уравнение из этого списка представляет собой уравнение КП в билинейной форме (2), в которой $u=2 \partial_{x}^{2} \ln \tau$.

Линейная задача КП (4) также может быть записана в билинейном виде $(\Phi=\rho / \sigma)$

$$
\operatorname{Res}_{\lambda}\left[\tau(\underline{t}-\underline{\epsilon}(\lambda)) \rho\left(\underline{t}^{\prime}+\underline{\epsilon}(\lambda)\right) e^{\xi\left(\underline{t}-\underline{t}^{\prime}, \lambda\right)}\right]=\rho(\underline{t}) \tau\left(\underline{t}^{\prime}\right)
$$

для любых $\underline{t}, \underline{t}^{\prime}$, или же с использованием оператора Хироты можно записать

$$
\sum_{j=0}^{\infty} p_{j}(-2 \underline{y}) p_{j}(\widetilde{D}) \exp \left(\sum_{i=1}^{\infty} y_{i} D_{i}\right) \rho \cdot \tau=\exp \left(\sum_{i=1}^{\infty} y_{i} D_{i}\right) \tau \cdot \rho
$$

для любого $\underline{y}$. Фундаментальное билинейное уравнение, содержашееся в этом бесконечном наборе, имеет вид

$$
\left(D_{2}-D_{1}^{2}\right) \rho \cdot \tau=0
$$

отвечающий линейному уравнению (5) при $\Phi=\rho / \sigma$ и $u=2 \partial_{x}^{2} \ln \tau$.

\section{3. ОПЕРАТОРЫ РЕКУРСИИ ДЛЯ РЕДУЦИРОВАННЫХ СИСТЕМ}

В этом разделе показывается, как можно вывести операторы рекурсии для размерно редуцированной иерархии КП непосредственно из билинейного тождества КП (7). Отметим сначала, что оператор рекурсии, вообще говоря, не существует для собственно $(2+1)$-мерного уравнения КП [9].

$k$-Редуцированная $(k=2,3, \ldots)$ иерархия задается условием симметрийной редукции $\tau_{t_{k}}=\lambda \tau$, где $\lambda$ - константа. Это условие подразумевает, что поля КП $u_{2}, u_{3}, \ldots$ не зависят от переменной $t_{k}$ (равно как и от переменных $t_{2 k}, t_{3 k}$ и т.д.). Условие редукции также можно выразить в терминах оператора Лакса иерархии КП: $L^{k}=B_{k}$. При $k=2$ получается иерархия Кд $\Phi$, а при $k=3$ - иерархия уравнения Буссинеска.

Вычисляя коэффициенты при членах $y_{m}$ в билинейных уравнениях КП (11), получим, что

$$
D_{1} D_{m} \tau \cdot \tau=2 p_{m+1}(\widetilde{D}) \tau \cdot \tau
$$


для любого $m$. Вычисляя коэффициент при $y_{m} y_{k}($ при $k \neq m$ ) в соотношении (11), получим уравнение

$$
\left(2 p_{m+k+1}(\widetilde{D})-p_{m+1}(\widetilde{D}) D_{k}-p_{k+1}(\widetilde{D}) D_{m}\right) \tau \cdot \tau=0 .
$$

Выразив первое слагаемое в этой формуле с помошью уравнения (16), получим

$$
\left(D_{1} D_{m+k}-p_{m+1}(\widetilde{D}) D_{k}-p_{k+1}(\widetilde{D}) D_{m}\right) \tau \cdot \tau=0 .
$$

В случае $k$-редуцированной иерархии КП, когда

$$
\tau_{t_{k}}=\lambda \tau,
$$

это соотношение принимает вид

$$
\left(D_{1} D_{m+k}-p_{k+1}(\widetilde{D}) D_{m}\right) \tau \cdot \tau=0 .
$$

Соотношение (20) задает каноническую билинейную форму $k$-редуцированной иерархии КП. Оно связывает поток $\tau_{t_{m}}$ с потоком $\tau_{t_{m+k}}$ и (будучи записанным как многочлен по производным $D_{1}, D_{2}, \ldots$ ) имеет фиксированную степень $k+2$, если $k$ четно, и $k+1$ при нечетных $k$ независимо от $m$.

Рассмотрим теперь случай $k=2$ (иерархия $\mathrm{Kд} \Phi$ ). В этом случае соотношение (20) имеет вид

$$
\left(D_{1} D_{m+2}-\left(\frac{D_{3}}{3}+\frac{D_{1} D_{2}}{2}+\frac{D_{1}^{3}}{6}\right) D_{m}\right) \tau \cdot \tau=0,
$$

или

$$
\left(D_{1} D_{m+2}-\frac{1}{3} D_{3} D_{m}-\frac{1}{6} D_{1}^{3} D_{m}\right) \tau \cdot \tau=0 .
$$

При $m=1$ это соотношение переходит в билинейное уравнение Кд $\Phi$

$$
\left(4 D_{1} D_{3}-D_{1}^{4}\right) \tau \cdot \tau=0,
$$

которое, в свою очередь, будучи записанным через поле $q=2 \ln \tau$, принимает вид

$$
4 q_{x, t_{3}}=q_{4 x}+3 q_{2 x}^{2} .
$$

Для $u=q_{2 x}$ получаем

$$
4 u_{t_{3}}=u_{3 x}+6 u u_{x} .
$$

При произвольном $m$ соотношение (22) представляет собой рекурсионное соотношение для иерархии $\mathrm{K}$ д :

$$
q_{x, t_{m+2}}-\frac{1}{3} q_{t_{3}, t_{m}}-\frac{1}{6} q_{3 x, t_{m}}-\frac{1}{2} q_{2 x} q_{x, t_{m}}=0 .
$$

Вычисляя $x$-производную этого соотношения и применяя уравнение $\mathrm{K} д \Phi(24)$, получим соотношение

$$
u_{t_{m+2}}=\left(\frac{1}{4} \partial_{x}^{2}+u+\frac{1}{2} u_{x} \partial_{x}^{-1}\right) u_{t_{m}}
$$

5 Теоретическая и математическая физика, т. 133, № 2, 2002 г. 
для поля Кд $\Phi=q_{2 x}=2 \partial_{x}^{2} \ln \tau$. Здесь используются обозначения $\partial_{x}^{-1} \equiv \int_{-\infty}^{x}$ и предполагается, что поле $u$ (и его производные) убывают на бесконечности достаточно быстро, чтобы обеспечить, например, $\partial_{x}^{-1} u_{x}=u$. Оператор $\partial_{x}^{2} / 4+3 u / 4-u_{x} \partial_{x}^{-1} / 2$ представляет собой хорошо известный оператор рекурсии Ленара для иерархии $\mathrm{Kд} \Phi$ [10], который может быть использован для порождения всех потоков старших порядков (симметрий) иерархии $\mathrm{K} д \Phi$, начиная с потока $u_{t_{1}}=u_{x}$. Если применить этот оператор к

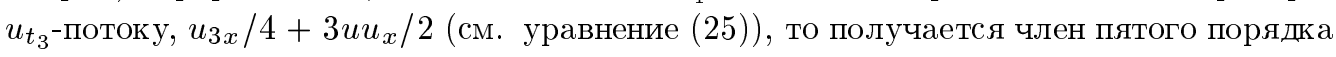
иерархии КдФ:

$$
u_{t_{5}}=\frac{1}{16} u_{5 x}+\frac{5}{8} u u_{3 x}+\frac{5}{4} u_{x} u_{2 x}+\frac{15}{8} u^{2} u_{x}
$$

где должны выполняться условия $\left(u_{t_{3}}\right)_{t_{5}}=\left(u_{t_{5}}\right)_{t_{3}}$ и т.д. Далее, оператор рекурсии задает сохраняющиеся плотности уравнения $\mathrm{K} д \Phi$ : оператор $\partial_{x}^{2} / 4+u-\partial_{x}^{-1} u_{x} / 2$, сопряженный оператору рекурсии, отображает сохраняюшиеся коварианты в сохраняюшиеся коварианты [4]. Например, сохраняюшаяся плотность $u$ отвечает сохраняюшемуся коварианту 1 , который отображается на $u$ сопряженным оператором рекурсии Кд $\Phi$. Этот сохраняюшийся ковариант отвечает сохраняющейся плотности $u^{2}$ и закону сохранения

$$
\left(u^{2}\right)_{t_{3}}=\frac{1}{2} u\left(u_{3 x}+6 u u_{x}\right)=\frac{1}{2}\left(u u_{2 x}-\frac{1}{2} u_{x}^{2}+2 u^{3}\right)_{x} .
$$

Второй пример - это 3-редуцированная иерархия КП (иерархия уравнения Буссинеска). Основное уравнение этой иерархии получается из уравнения КП (12), если положить $\tau_{t_{3}}=\lambda \tau$ :

$$
\left(3 D_{2}^{2}+D_{1}^{4}\right) \tau \cdot \tau=0,
$$

или же, в терминах полей $u=q_{2 x}=2 \partial_{x}^{2} \ln \tau$ и $v=q_{x, t_{2}}=2 \partial_{x} \partial_{t_{2}} \ln \tau$, получим

$$
\begin{aligned}
& u_{t_{2}}=v_{x} \\
& v_{t_{2}}=-\frac{1}{3} u_{3 x}-2 u u_{x} .
\end{aligned}
$$

Записанная в таком виде система иногда называется двухкомпонентным уравнением Буссинеска. Билинейная форма (20) при $k=3$ принимает вид

$$
\left(D_{1} D_{m+3}-p_{4}(\widetilde{D}) D_{m}\right) \tau \cdot \tau=0,
$$

или

$$
\left(D_{1} D_{m+3}-\frac{1}{4} D_{1}^{2} D_{2} D_{m}-\frac{1}{4} D_{4} D_{m}\right) \tau \cdot \tau=0 .
$$

Это каноническая билинейная форма иерархии Буссинеска. Второй член в иерархии Буссинеска НУрЧП получается, если в этом выражении положить $m=1$ :

$$
q_{x, t_{4}}=\frac{1}{3} q_{3 x, t_{2}}+q_{2 x} q_{x, t_{2}},
$$


или же, в терминах полей $u$ и $v$,

$$
\begin{aligned}
& u_{t_{4}}=\frac{1}{3} v_{3 x}+(u v)_{x}, \\
& v_{t_{4}}=-\frac{1}{9} u_{5 x}-2 u_{x} u_{2 x}-u u_{3 x}+v v_{x}-2 u^{2} u_{x} .
\end{aligned}
$$

При произвольном значении $m$ каноническая билинейная форма уравнения Буссинеска имеет вид

$$
q_{x, t_{m+3}}-\frac{1}{4}\left(q_{2 x, t_{2}, t_{m}}+q_{2 x} q-t_{2}, t_{m}+2 q_{x, t_{2}} q_{x, t_{m}}\right)-\frac{1}{4} q_{t_{4}, t_{m}}=0 .
$$

Производная по $x$ от этого соотношения дает формулу

$$
u_{t_{m+3}}-\frac{1}{3} v_{2 x, t_{m}}-\frac{1}{4} u_{x} \partial_{x}^{-1} v_{t_{m}}-\frac{1}{2} u v_{t_{m}}-\frac{3}{4} v u_{t_{m}}-\frac{1}{2} v_{x} \partial_{x}^{-1} u_{t_{m}}=0,
$$

где также надо использовать первое уравнение системы (33). Производная соотношения (35) по $t_{2}$ дает

$$
\begin{gathered}
v_{t_{m+3}}-\frac{1}{4}\left(-\frac{1}{3} u_{2 x}-u^{2}\right)_{2 x, t_{m}}-\frac{1}{4} v_{x} \partial_{x}^{-1} v_{t_{m}}-\frac{1}{4} u\left(-\frac{1}{3} u_{2 x}-u^{2}\right)_{t_{m}}- \\
-\frac{1}{2}\left(-\frac{1}{3} u_{2 x}-u^{2}\right)_{x} \partial_{x}^{-1} u_{t_{m}}-\frac{1}{2} v v_{t_{m}}-\frac{1}{4} \partial_{x}^{-1}\left(v_{t_{4}}\right)_{t_{m}}=0
\end{gathered}
$$

где надо использовать основное уравнение Буссинеска (29) для того, чтобы убрать член $q_{2 t_{2}}$, и второе уравнение системы $(34)$ для того, чтобы убрать член $v_{t_{4}}$. Уравнения (36) и (37) могут быть представлены в виде

$$
\left(\begin{array}{l}
u \\
v
\end{array}\right)_{t_{m+3}}=\left(\begin{array}{ll}
R_{11} & R_{12} \\
R_{21} & R_{22}
\end{array}\right)\left(\begin{array}{l}
u \\
v
\end{array}\right)_{t_{m}},
$$

где

$$
\begin{aligned}
& R_{11}=\frac{3}{4} v+\frac{1}{2} v_{x} \partial_{x}^{-1} \\
& R_{12}=\frac{1}{2} \partial_{x}^{2}+\frac{1}{2} u+\frac{1}{4} u_{x} \partial_{x}^{-1}, \\
& R_{21}=-\frac{1}{9} \partial_{x}^{4}-\frac{5}{4} u_{x} \partial_{x}-\frac{3}{4} u_{2 x}-\frac{5}{6} u \partial_{x}^{2}-u^{2}-\frac{1}{2}\left(\frac{1}{3} u_{3 x}+2 u u_{x}\right) \partial_{x}^{-1}, \\
& R_{22}=\frac{1}{4} v_{x} \partial_{x}^{-1}+\frac{3}{4} v .
\end{aligned}
$$

Эта форма записи представляет собой (матричный) оператор рекурсии для уравнения Буссинеска (см. [4], с.323). Его формально сопряженньй оператор опять может быть использован для построения любого числа законов сохранения в этой иерархии.

В качестве третьего примера рассмотрим 1-ограниченную иерархию КП. Легко показать, что $\left(\Phi \Phi^{\star}\right)_{x}$ является симметрией уравнения КП $(2)$, если $\Phi$ и $\Phi^{*}$ суть собственные функции линейных уравнений КП (5) и их сопряженных. При этом можно наложить условие симметрийной редукции $u_{x}=\left(\Phi \Phi^{\star}\right)_{x}$, чтобы привести иерархию КП к 
$(1+1)$-мерной иерархии. Основное свойство этой редукции состоит в том, что производная тау-функции КП по $x$ опять оказывается тау-функцией КП [11], [12]. Следовательно, если иерархия КП содержит билинейное уравнение $B \tau \cdot \tau=0$, то 1-ограниченная иерархия КП содержит дополнительно уравнение $B \tau_{x} \cdot \tau_{x}=0$, или, эквивалентно, $D_{x}^{2} B \tau \cdot \tau=0$ (так как $\left.\partial_{x}^{2}(B \tau \cdot \tau)-4 B \tau_{x} \cdot \tau_{x}=D_{x}^{2} B \tau \cdot \tau\right)$. Поэтому некоторые из билинейных уравнений этой иерархии имеют вид

$$
\left(4 D_{x} D_{t_{3}}-3 D_{t_{2}}^{2}-D_{x}^{4}\right) \tau \cdot \tau=0, \quad\left(4 D_{x}^{3} D_{t_{3}}-3 D_{x}^{2} D_{t_{2}}^{2}-D_{x}^{6}\right) \tau \cdot \tau=0
$$

и т.д. 1-ограниченная иерархия КП также известна под именем нелокальной иерархии Буссинеска и оказывается тесно связанной с нелинейным уравнением Шредингера. Уравнение низшего порядка в этой иерархии имеет вид

$$
\begin{aligned}
& u_{t_{2}}=v_{x} \\
& v_{t_{2}}=u_{3 x}+\left(u^{2}\right)_{x}+\left[\frac{v^{2}-u_{x}^{2}}{u}\right]_{x},
\end{aligned}
$$

где опять $u=q_{2 x}$ и $v=q_{x, t_{2}}$. Это уравнение имеет те же члены линейной дисперсии, что и уравнение Буссинеска (30), но нелинейности этих уравнений отличаются. 1-ограниченная иерархия КП содержит билинейные соотношения

$$
D_{1}^{3} D_{m} \tau \cdot \tau=2 p_{m+1}(\widetilde{D}) D_{1}^{2} \tau \cdot \tau
$$

для любого $m$. Коэффициент при члене $y_{m} y_{1}^{2}$ в билинейном тождестве КП (11) имеет вид

$$
\begin{gathered}
\left(-4 p_{m+3}(\widetilde{D})+4 p_{m+2}(\widetilde{D}) D_{1}-p_{m+1}(\widetilde{D}) D_{1}^{2}+2 p_{3}(\widetilde{D}) D_{m}-\right. \\
\left.-2 p_{2}(\widetilde{D}) D_{1} D_{m}+\frac{1}{2} D_{1}^{3} D_{m}\right) \tau \cdot \tau=0
\end{gathered}
$$

Если выразить член $p_{m+2}(\widetilde{D}) D_{1} \tau \cdot \tau$ из соотношения $(18)$ (при $k=1$ ), а член $p_{m+1}(\widetilde{D}) D_{1}^{2} \tau \cdot \tau$ из соотношения $(42)$, то в результате получим

$$
\left(3 D_{1} D_{m+2}-3 D_{2} D_{m+1}-D_{1}^{3} D_{m}+D_{3} D_{m}\right) \tau \cdot \tau=0 .
$$

Эта формула содержит потоки $t_{m}, t_{m+1}$ и $t_{m+2}$; с ее помощью можно породить все уравнения этой иерархии, начиная с потока $t_{1}=x$ и основного уравнения (41). Заметим, что случаи $m=1$ и $m=2$ задают билинейные уравнения, содержашиеся в нередуцированной иерархии КП (см. (12)), но уже билинейное уравнение при $m=3$ в ней не содержится: никаких канонических билинейных форм для иерархии КП не существует. Оператор рекурсии для этой иерархии имеет вид [12]

$$
\mathcal{R}(u, v)=\left(\begin{array}{cc}
-\frac{v}{2 u}-\frac{u_{x}}{2} \partial_{x}^{-1} \frac{v}{u^{2}} & 1+\frac{u_{x}}{2} \partial_{x}^{-1} \frac{1}{u} \\
\partial_{x}^{2}+2 u-\frac{3 u_{x}}{2 u} \partial_{x}- & \frac{3 v}{2 u}+\frac{v_{x}}{2} \partial_{x}^{-1} \frac{1}{u} \\
-\frac{u_{2 x}}{2 u}-\frac{v^{2}-u_{x}^{2}}{u^{2}}-\frac{v_{x}}{2} \partial_{x}^{-1} \frac{v}{u^{2}} &
\end{array}\right)
$$


Как предполагалось выше, с каждым потоком, порожденным $R$, можно ассоциировать эволюционирующую систему

$$
\left(\begin{array}{l}
u \\
v
\end{array}\right)_{t_{m+1}}=\mathcal{R}(u, v)\left(\begin{array}{l}
u \\
v
\end{array}\right)_{t_{m}}
$$

по переменным $t_{1}=x, t_{2}=t, t_{3}, \ldots$ Применяя оператор (45) к тривиальной симметрии $\left(u_{x}, v_{x}\right)$, получим нелинейный поток Буссинеска (41). Первый поток старшего порядка имеет вид

$$
\begin{aligned}
& u_{t_{3}}=u_{3 x}+\frac{3}{2}\left(u^{2}\right)_{x}+\frac{3}{4}\left[\frac{-v^{2}+u_{x}^{2}}{u}\right]_{x}, \\
& v_{t_{3}}=-v_{3 x}-3(u v)_{x}+\frac{3}{2}\left[\frac{v_{x} u_{x}-v u_{2 x}}{u}\right]_{x}+\frac{3}{4}\left[\frac{-v^{3}+u_{x}^{2} v}{u^{2}}\right]_{x} .
\end{aligned}
$$

Можно показать, что оператор рекурсии (45) "наследуется", а потому сушествует бесконечная последовательность согласованных уравнений. Из этого рекурсионного соотношения следуют канонические билинейные соотношения (44). Некоторые из сохраняющихся плотностей этого уравнения имеют вид

$$
u, \quad v, \quad \frac{u^{2}}{2}+\frac{1}{4} \frac{v^{2}-u_{x}^{2}}{u}, \ldots .
$$

\section{4. ПАРЫ ЛАКСА ДЛЯ РЕДУЦИРОВАННЫХ ИЕРАРХИЙ}

В этом разделе будет показано, как вьводить (аналогично разделу 3) канонические пары Лакса для редуцированных уравнений КП. Начнем с модифицированного билинейного тождества КП (14), в котором коэффициент при $y_{m}$ имеет вид

$$
\left(p_{m}(\widetilde{D})-D_{m}\right) \rho \cdot \tau=0
$$

для любого $m$, в то время как коэффициент при $y_{m} y_{k}$ дает

$$
\left(-2 p_{m+k}(\widetilde{D})+p_{m}(\widetilde{D}) D_{k}+p_{k}(\widetilde{D}) D_{m}-D_{m} D_{k}\right) \rho \cdot \tau=0
$$

Используем сначала соотношение (47) для того, чтобы убрать первый член в выражении (48). В случае $k$-редуцированной иерархии $\left(\tau_{t_{k}}=\lambda_{1} \tau\right.$ и $\left.\rho_{t_{k}}=\lambda_{2} \rho\right)$ можно просто положить $D_{k} \rightarrow \lambda$, а потом заменить $\lambda p_{m}(\widetilde{D})$ с помошью формулы $(47)$ :

$$
\left(-2 D_{m+k}+p_{k}(\widetilde{D}) D_{m}\right) \rho \cdot \tau=0 .
$$

Степень этой формы Хироты равна $k+1$ независимо от $m$. Она представляет собой каноническую билинейную форму модифицированной $k$-редуцированной иерархии КП.

Например, при $k=2$ (случай иерархии КдФ) найдем, что

$$
\left(-2 D_{m+2}-\frac{\lambda}{4} D_{m}-\frac{1}{4} D_{1}^{2} D_{m}\right) \rho \cdot \tau=0 .
$$


В случае $m=1$ это соотношение приводит к формуле

$$
\left(D_{t_{3}}-\frac{\lambda}{4} D_{x}-\frac{1}{4} D_{x}^{3}\right) \rho \cdot \tau=0
$$

Делая замену переменных $\psi=\rho / \tau$ и $u=2 \partial_{x}^{2} \ln \tau$, получим

$$
\psi_{t_{3}}-\frac{\lambda}{4} \psi_{x}-\frac{1}{4}\left(\psi_{3 x}+3 u \psi_{x}\right)=0
$$

Условие совместности этого линейного уравнения (уравнения Лакса) с задачей на собственные значения, получающейся из условий (5) и линейной задачи $\psi_{t_{2}}=\lambda \psi$,

$$
\psi_{2 x}+u \psi=\lambda \psi
$$

порождает уравнение $\mathrm{Kд} \Phi 4 u_{t_{3}}-u_{3 x}-6 u u_{x}=0$, если только $\lambda_{t_{3}}=0$.

Благодарности. Работа получила финансовую поддержку от Федерального министерства науки, техники и культуры при Администрации премьер-министра Бельгии (контракт № P4/08 “Interuniversity poles of attraction programme”). Автор является post-doctoral fellow at the Fwo-Vlaanderen (Belgium).

\section{Список литературы}

[1] D. J. Korteweg, G. de Vries. Philos. Mag. Ser. 5. 1895. V. 39. P. 422-443.

[2] J. Boussinesq. C. R. Acad. Sci. Paris. 1871. V. 72. P. 755-759.

[3] M. Remoissenet. Waves Called Solitons: Concepts and Experiments. Berlin: Springer, 1994.

[4] П. Олвер. Приложение групп Ли к дифференциальным уравнениям. М.: Мир, 1989.

[5] Р. Хирота. Прямые методы в теории солитонов. В сб.: Солитоны. Ред. Р. Буллаф, Ф. Кодри. М.: Мир, 1983. С. 175-192.

[6] Б. Б. Кадомцев, В. И. Петвиашвили. ДАН СССР. 1970. Т. 192. С. 753-756.

[7] R. Willox. On a direct bilinear operator method in soliton theory Ph.D. thesis Vrije Universiteit Brussel Brussels. Belgium, 1993.

[8] E. Date, M. Kashiwara, M. Jimbo, T. Miwa. Transformation groups for soliton equations. In: Proceedings of RIMS symposium on non-linear integrable systems-Classical theory and quantum theory. Eds. M. Jimbo, T. Miwa. Singapore: World Scientific, 1983. P. 39-119.

[9] A.S. Fokas, P. M. Santini. Commun. Math. Phys. 1988. V. 116. P. 449-474.

[10] B. Fuchssteiner. Nonlinear Anal. Theor. Meth. Appl. 1979. V. 6. P. 849-862.

[11] F. Lambert, I. Loris, J. Springael, R. Willox. J. Phys. A. 1994. V. 27. P. 5325-5334; I. Loris, R. Willox. Inverse Problems. 1997. V. 13. P. 411-420; I. Loris, R. Willox. J. Phys. A. 1997. V. 30. P. 6925-6938.

[12] I. Loris. Symmetry reductions in the tau-function approach to integrability Ph.D. thesis Vrije Universiteit Brussel Brussels. Belgium, 1998. 